03

\title{
Взаимодействие осциллонов конденсата Бозе-Эйнштейна
}

\author{
(C) Н.В. Высотина ${ }^{1}$, Н.Н. Розанов ${ }^{1,2,3, \uparrow}$, А.Н. Шацев ${ }^{1}$ \\ ${ }^{1}$ Государственный оптический институт им. С.И. Вавилова, \\ 199053 Санкт-Петербург, Россия \\ ${ }^{2}$ Университет ИТМО, \\ 197101 Санкт-Петербург, Россия \\ ${ }^{3}$ Физико-технический институт им. А.Ф. Иофффе, \\ 194021 Санкт-Петербург, Россия \\ ฯ e-mail: nnrosanov@mail.ru
}

Поступила в редакцию 04.09.2017 г.

Численно промоделировано взаимодействие двух одномерных пространственных векторных локализованных структур атомного конденсата Бозе-Эйнштейна в ловушке со значительными поперечными размерами и осциллирующими стенками. Выявлены различные типы связанных структур и различные сценарии их столкновений. Проводится сравнение со свойствами скалярных солитонов, описываемых традиционным нелинейным уравнением Шредингера.

DOI: $10.21883 /$ OS.2018.01.45363.193-17

\section{Введение}

Локализованные структуры атомного бозе-эйнштейновского конденсата (БЭК) в динамической ловушке (с осциллирующими стенками), которые могут быть перспективными для прецизионных измерений, занимают промежуточное место между консервативными и диссипативными солитонами. Это вызвано тем, что в таких системах в случае непрозрачных для атомов стенок сохраняется число частиц, но, с другой стороны, имеется энергообмен при взаимодействии атомов с движущимися стенками $[1,2]$. В резонансных условиях, когда частота осцилляций стенок близка к разности частот какой-либо пары стационарных состояний БЭК и соответственно оправдано двухуровневое приближение, динамика БЭК описывается системой связанных уравнений типа нелинейных уравнений Шредингера (НУШ) [1-5]. Подобные векторные (в отличие от скалярных, описываемых единственным НУШ) солитоны фигурируют в широком круге нелинейных систем (например, [6]), но только в исключительных случаях возможно аналитическое решение соответствующей системы НУШ [7]. Спецификой системы уравнений для солитонов БЭК в ловушке с осциллирующими стенками, которые мы будем называть осциллонами, служит одновременное присутствие линейной когерентной и нелинейной некогерентной связи (когерентность здесь указывает на чувствительность к фазовым соотношениям). В общем случае нахождение аналитического решения соответствующей системы уравнений не представляется возможным, что подчеркивает значимость численного моделирования в этих задачах.

Некоторые результаты численных расчетов характеристик осциллонов БЭК приведены в обзорах [1,2]. Задачей настоящего сообщения служит более детальный анализ взаимодействия двух одномерных пространственных векторных осциллонов для ловушки со значительными поперечными размерами. В следующем разделе приведены исходные уравнения и соотношения. Основное внимание уделяется специальному типу векторных солитонов - так называемым синфазным и противофазным осциллонам, поскольку для них более четко прослеживаются соотношения со скалярными солитонами. Далее рассматриваются взаимодействия двух осциллонов, приводящие к формированию их связанных структур. Затем рассматриваются различные сценарии столкновений двух осциллонов. Работа завершается общим обсуждением результатов.

\section{Модель и управляющие уравнения}

Моделью служит БЭК холодного (при температуре ниже критической для существования БЭК) разреженного газа слабо взаимодействующих атомов в сигарообразной ловушке с осциллирующими стенками. В приближении среднего поля динамика БЭК описывается уравнением Гросса-Питаевского для макроскопической волновой функции БЭК $\psi[8]$ :

$$
i \frac{\partial \psi}{\partial t}=-\nabla^{2} \psi+U \psi+U_{0}|\psi|^{2} \psi
$$

Здесь $t$ - время, $\nabla^{2}=\partial^{2} / \partial x^{2}+\partial^{2} / \partial y^{2}+\partial^{2} / \partial z^{2}-$ оператор Лапласа, $x, y, z$ - декартовы координаты, $U-$ потенциал ловушки, $U_{0}= \pm 1-$ знак постоянной взаимодействия атомов; уравнение записано для безразмерных переменных. Аналогичное уравнение для конденсата экситонов или поляритонов в полупроводниках известно как уравнение Келдыша [9]. Уравнением (1) описывается и родственная схема с БЭК в поле тяготения [10-12].

Считаем, что стенки ловушки, расположенные при $z=L_{\text {left }}(t)$ и $L_{\text {right }}(t)$, где $z$ - продольная координата вдоль оси ловушки, непрозрачны для атомов. Тогда граничные условия к уравнению (1) следующие:

$$
\psi\left(z=L_{\text {left }}(t)\right)=\psi\left(z=L_{\text {right }}(t)\right)=0 .
$$


Будем полагать, что осцилляции стенок гармонические с частотой $\Omega$ и малой глубиной модуляции $\mu$; для определенности считаем левое зеркало неподвижным:

$$
L_{\text {left }}(t)=0, \quad L_{\text {right }}(t)=L_{0}(1+\mu \cos \Omega t) .
$$

При гармонических осцилляциях и слабой нелинейности полезным является понятие квазиэнергии и определение ее спектра $[3,4]$. Поскольку поперечное распределение потенциала для идеальных стенок (2) отвечает прямоугольной квантовой яме, то спектр квазиэнергии существенно неэквидистантен. Поэтому при частоте осцилляций стенок, близкой к разности частот пары квазиэнергетических состояний, реализуется резонансное взаимодействие между этими состояниями с амплитудами $a_{0}$ и $a_{1}$ с соответствующими населенностями $n_{0,1}=\left|a_{0,1}\right|^{2}$. Для поперечно широкой ловушки безразмерные управляющие уравнения принимают [1-4] следующий вид:

$$
\begin{aligned}
& i \frac{\partial a_{0}}{\partial t}+\frac{\partial^{2} a_{0}}{\partial x^{2}}+a_{1}+\left(3\left|a_{0}\right|^{2}+4\left|a_{1}\right|^{2}\right) a_{0}=0 \\
& i \frac{\partial a_{1}}{\partial t}+\frac{\partial^{2} a_{1}}{\partial x^{2}}+a_{0}+\left(3\left|a_{1}\right|^{2}+4\left|a_{0}\right|^{2}\right) a_{1}=0 .
\end{aligned}
$$

Здесь мы ограничились случаем точного резонанса, планарной ловушки $\left(\nabla_{\perp}^{2} \rightarrow \partial^{2} / \partial x^{2}, x-\right.$ поперечная координата) и $U_{0}=-1$ (притяжение атомов, аналог самофокусировочной нелинейности). Уравнения (4) описывают двухкомпонентную (векторную) систему с линейным когерентным и нелинейным некогерентным взаимодействиями компонент. Квадрат модуля амплитуд определяет населенности резонансных уровней $n_{0,1}=\left|a_{0,1}\right|^{2}$, причем общее число частиц в ловушке, согласно (4), сохраняется:

$$
\frac{d N}{d t}=0, \quad N=\int\left(\left|a_{0}\right|^{2}+\left|a_{1}\right|^{2}\right) d x
$$

Такой закон сохранения справедлив ввиду идеальной непроницаемости стенок ловушки. Если стенки отвечают потенциальному барьеру конечной глубины, то атомы с конечной вероятностью ускользают из ловушки и время жизни конденсата становится ограниченным [13].

Введем поперечную координату центра локализованной структуры интегральным соотношением:

$$
x_{c}(t)=\int_{-\infty}^{+\infty} x\left(\left|a_{1}\right|^{2}+\left|a_{0}\right|^{2}\right) d x / N
$$

Используя (4), можно показать, что (поперечная) скорость центра структуры $v_{c}=d x_{c} / d t$ сохраняется:

$$
\frac{d v_{c}}{d t}=\frac{d^{2} x_{c}}{d t^{2}}=0
$$

Уравнения (4) инвариантны к сдвигу поперечной координаты $x$ и обладают галилеевой симметрией. Последнее означает, что если имеется решение $(5)$ вида $a_{0,1}^{(0)}(x, t)$, то имеется и семейство решений вида [14]

$$
a_{0,1}(x, t)=a_{0,1}^{(0)}(x-V t, t) \exp \left(i \frac{V}{2} x-i \frac{V^{2}}{4} t\right)
$$

с произвольной поперечной скоростью $V$.

В принятом случае точного резонанса система (4) обладает дополнительной симметрией относительно замены $a_{0,1} \leftrightarrow a_{1,0}$. Ввиду этого имеются синфазные $\left(a_{0}=a_{1}\right)$ и противофазные $\left(a_{0}=-a_{1}\right)$ решения (4), для которых система (4) сводится к единственному уравнению:

$$
i \frac{\partial a}{\partial t}+\nabla_{\perp}^{2} a \pm a+7|a|^{2} a=0 .
$$

После замены $a=b e^{ \pm i t}$ из (9) следует классическое НУШ:

$$
i \frac{\partial b}{\partial t}+\nabla_{\perp}^{2} b+7|b|^{2} b=0 .
$$

Нелинейное уравнение Шредингера решается методом обратной задачи рассеяния $[14,15]$, и для него известны семейства солитонных решений вида

$$
a=\sqrt{2 \Omega_{0} / 7} \operatorname{ch}^{-1}\left(\sqrt{\Omega_{0}} x\right) e^{i\left(\Omega_{0} \pm 1\right) t} .
$$

Параметр $\Omega$ определяет частотный сдвиг, ширину $w=1 / \sqrt{\Omega_{0}}$ и максимальную амплитуду солитона. При задании начального профиля амплитуд вида (11), но с отличающейся максимальной амплитудой, происходит возврат к идеальной форме (11) за времена порядка нескольких тысяч безразмерных единиц для использованных в расчетах параметров. Помимо одиночных солитонов, известны устойчивые многосолитонные решения НУШ, а также бризеры - осциллирующие локализованные структуры, которые можно рассматривать как связанные состояния солитонов и которые обладают при наличии малых возмущений большим временем жизни. Отметим тем не менее, что характер устойчивости локализованных структур для скалярного НУШ и векторных осциллонов системы уравнений (4) может различаться, ввиду чего требуются дополнительные исследования применительно к (4). В частности, светлые одиночные осциллоны в отличие от солитонов НУШ обладают долгоживущими внутренними модами [2]. Далее мы приведем результаты численного решения системы (4) с различными начальными условиями, отвечающими взаимодействию двух осциллонов.

\section{Связанные поперечные осциллоны}

Рассмотрим сначала динамику пары исходно неподвижных $\left(V_{1,2}=0\right)$ синфазных или противофазных осциллонов с профилем амплитуд компонент вида (11), разделенных расстоянием $\Delta x$. Частоты синфазных и противофазных осциллонов различны даже при $\Omega_{01}=\Omega_{02}$, а при $\Omega_{01} \neq \Omega_{02}$ различаются и максимальные амплитуды и соответственно ширины осциллонов. 

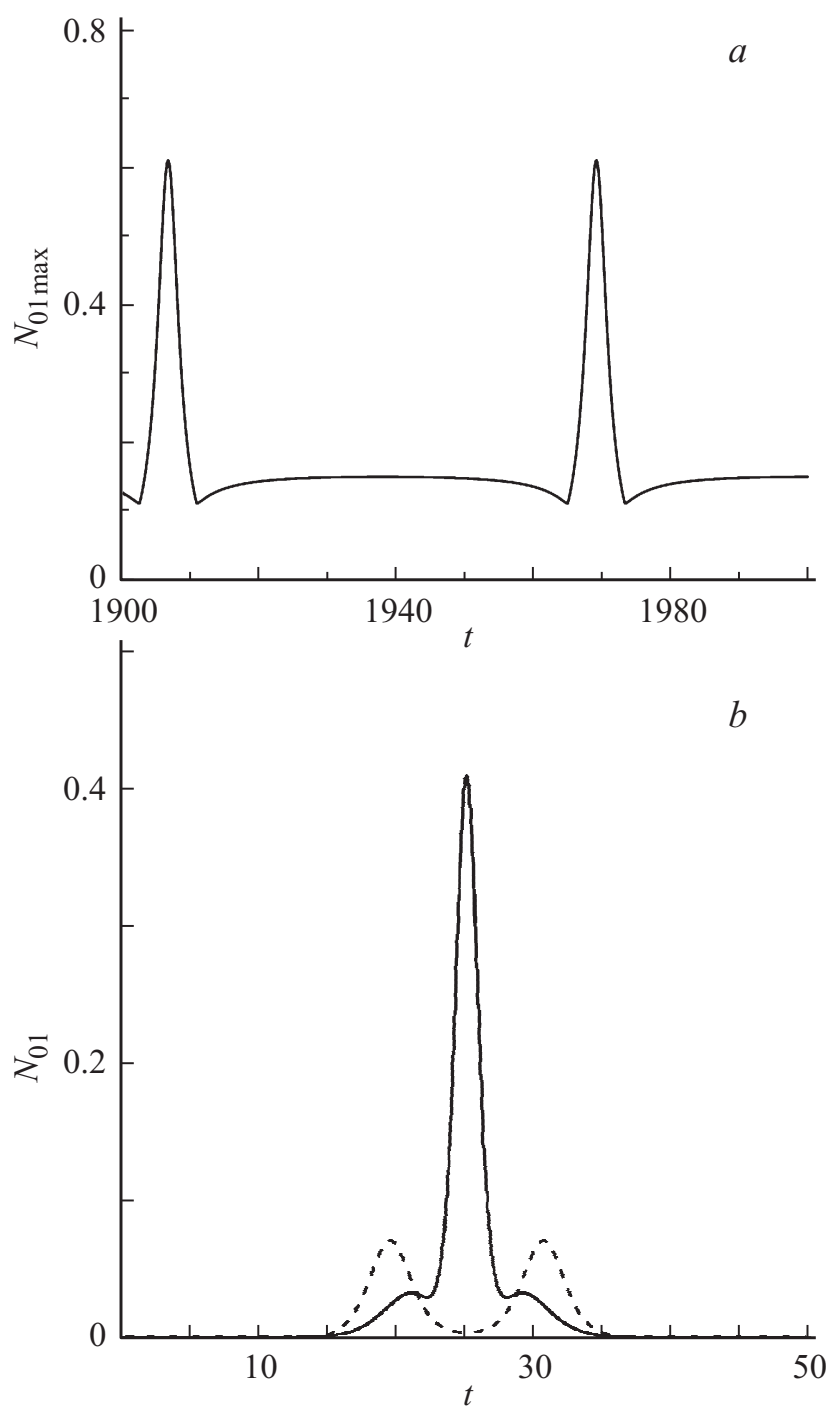

Рис. 1. Максимальная по поперечной координате $x$ суммарная населенность $N_{01}=\left|a_{0}\right|^{2}+\left|a_{1}\right|^{2}$ для векторного бризера, формирующегося из пары исходно неподвижных синфазных осциллонов с параметрами $\Omega_{01}=\Omega_{02}=0.25\left(w_{1}=w_{2}=2\right)$, разделенных интервалом $\Delta x=12:(a)$ временная зависимость максимальной населенности, $(b)$ профиль суммарной населенности в моменты времени $t=30(1), 50$ (2).

\section{Синфазные осциллоны}

На рис. 1 представлена симметричная структура, устанавливающаяся при больших временах (время установления увеличивается при уменьшении расстояния $\Delta x$ ) из пары одинаковых синфазных осциллонов. Эта структура описывает периодические временные пульсации как самого профиля суммарной населенности (двугорбые профили чередуются с одногорбыми или в течение коротких промежутков времени - с трехгорбыми), так и максимальной по координате населенности. В данном случае при всех временах (время расчета превышает 15000) выполняется соотношение $\left|a_{0}(x, t)\right|^{2}=\left|a_{1}(x, t)\right|^{2}$, ввиду чего система (4) сводится к единственному скалярно- му НУШ. Поэтому иллюстрируемую рис. 1 структуру можно считать бризером. Естественно, что период и амплитуда колебаний структуры зависят от ширины $w$ (максимальной амплитуды) исходных осциллонов и расстояния между ними $\Delta x$ (с ростом этих величин период возрастает).

Для асимметричной пары синфазных осциллонов система (4) уже не сводится к единственному НУШ, т.е. здесь проявляется дополнительная (векторная) степень свободы. На рис. 2 приведен пример при двукратном отличии начальных ширин осциллонов. На рис. 2, $a, b$ показана траектория максимума общей населенности $x_{\max }(t)$, которая в отличие от предыдущего варианта осциллирует с двумя существенно различающимися характерными масштабами периода (61 и 0.4). Кроме того, на рис. 2, a можно отметить небольшую постоянную составляющую скорости. Это не противоречит приведенному выше соотношению (7), которое свидетельствует о сохранении скорости движения интегрального центра, поскольку здесь говорится о другой величине - скорости перемещения максимума населенности. Осциллируют и населенности уровней (рис. 2,c). При больших временах населенности уровней выравниваются:

$$
\left|a_{0}(x, t)\right|^{2} \approx\left|a_{1}(x, t)\right|^{2} .
$$

Поэтому система (4) приближенно сводится к единственному НУШ. Однако в отличие от предыдущего варианта структура остается выраженно асимметричной, причем в двугорбых структурах главный максимум населенности находится то с одной, то с другой стороны от второго максимума (рис. $2, d$ ). Тем самым здесь реализуется долгоживущий резко асимметричный бризер.

\section{Пара из синфазного и противофазного осциллонов}

По-отдельности такие осциллоны обладают различающейся частотой, поэтому в данном случае управляющие уравнения не сводятся к единому НУШ и полностью проявляется векторная природа структуры. Даже если начальные распределения населенностей компонент совпадают (профили пространственно совмещены), то со временем эти профили начинают расходиться, что иллюстрирует рис. 3. Примечательно поведение максимума населенности (рис. 3,a,c). До $t=8000$ профили населенностей в осциллонах практически неподвижны. Однако при $t>8000$ профили перестраиваются с отсеиванием волн сплошного спектра, и начинается однонаправленное движение этой связанной структуры с постоянной скоростью. Как уже указывалось, изменение скорости движения максимумов населенности не находится в противоречии с постоянством интегральной скорости движения.

На рис. 4 показана динамика пары исходно пространственно разделенных синфазного и противофазного осциллонов. Из рис. 4, $a$ видно, что исходно неподвижная 


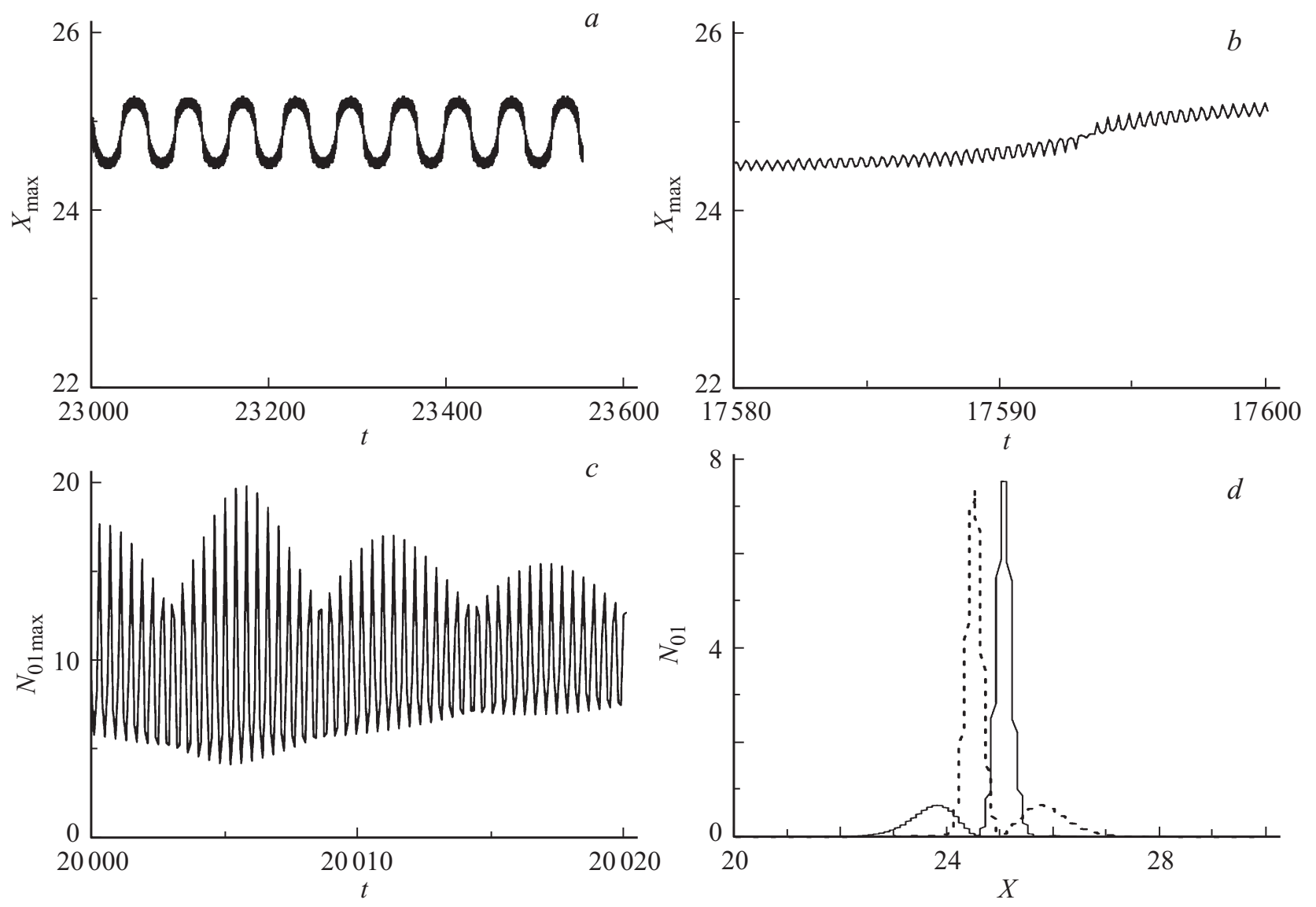

Рис. 2. Динамика асимметричной синфазной пары осциллонов: $(a),(b)$ - траектория максимума суммарной населенности в различных временных масштабах, $(c)$ - временные осцилляции максимальной суммарной населенности уровней, $(d)-$ профили населенности отдельного уровня $\left|a_{0}(x, t)\right|^{2} \approx\left|a_{1}(x, t)\right|^{2}$ в моменты времени $t=100$ (пунктир), 300 (сплошная кривая). Параметры: $w_{1}=0.66, w_{2}=0.33, \Delta x=1.4$.

структура после некоторого этапа перестройки начинает двигаться, причем максимум населенности приобретает постоянную скорость. Максимальная суммарная населенность $N_{01}$ каждого из осциллонов в паре приближается к постоянной величине (рис. $4, b$ ), тогда как населенности компонент $N_{0}$ и $N_{1}$ колеблются в противофазе (рис. $4, c)$.

\section{Столкновения осциллонов}

Рассмотрим теперь столкновение двух осциллонов, первоначально разведенных на расстояние, значительно превышающее их ширины, и потому не взаимодействующих. Соответственно профили населенности для каждого из солитонов задаются или соотношением (11) для неподвижного осциллона, или соотношением, вытекающим из (11) после преобразования Галилея (7), для осциллона, движущегося со скоростью $V$. Ввиду (7) сценарий столкновения зависит лишь от относительной скорости (разности исходных скоростей) осциллонов. Поэтому без ограничения общности можно считать один из двух осциллонов неподвижным, а другой - налетающим на него со скоростью $V$. В расчетах движущийся осциллон стартовал с координаты $x=0$, а неподвижный располагался при $x=50 \gg w \sim 1$.

При больших значениях скорости $V$ время столкновения $\tau \sim w / V$ мало, ввиду чего взаимодействие осциллонов будет слабым. Оценить условие реализации такого режима можно, потребовав малость нелинейного фазового набега за время столкновения, для которого из (4) находим: $\varphi_{n l} \sim 7\left|a_{0}\right|^{2} \tau \sim 2 /(w V)$. Требование $\varphi_{n l} \ll 1$ и дает условие для скорости: $V \gg 2 / w$. Это условие имеет универсальный характер, будучи применимым к широкому классу солитонов и солитонных комплексов различных типов.

В рассматриваемых вариантах число осциллонов при столкновении не меняется, в связи с чем возникает вопрос о том, возможно ли проследить судьбу индивидуальных осциллонов. В общем случае сделать это затруднительно ввиду невозможности ввести четкие критерии различения осциллонов. При этом, по-видимому, имеются два исключения, в которых индивидуальность осциллонов прослеживается естественным образом. Первый относится к сценариям, в которых осциллоны только сближаются до определенного расстояния, после чего расходятся из-за отталкивания. Тогда можно говорить 


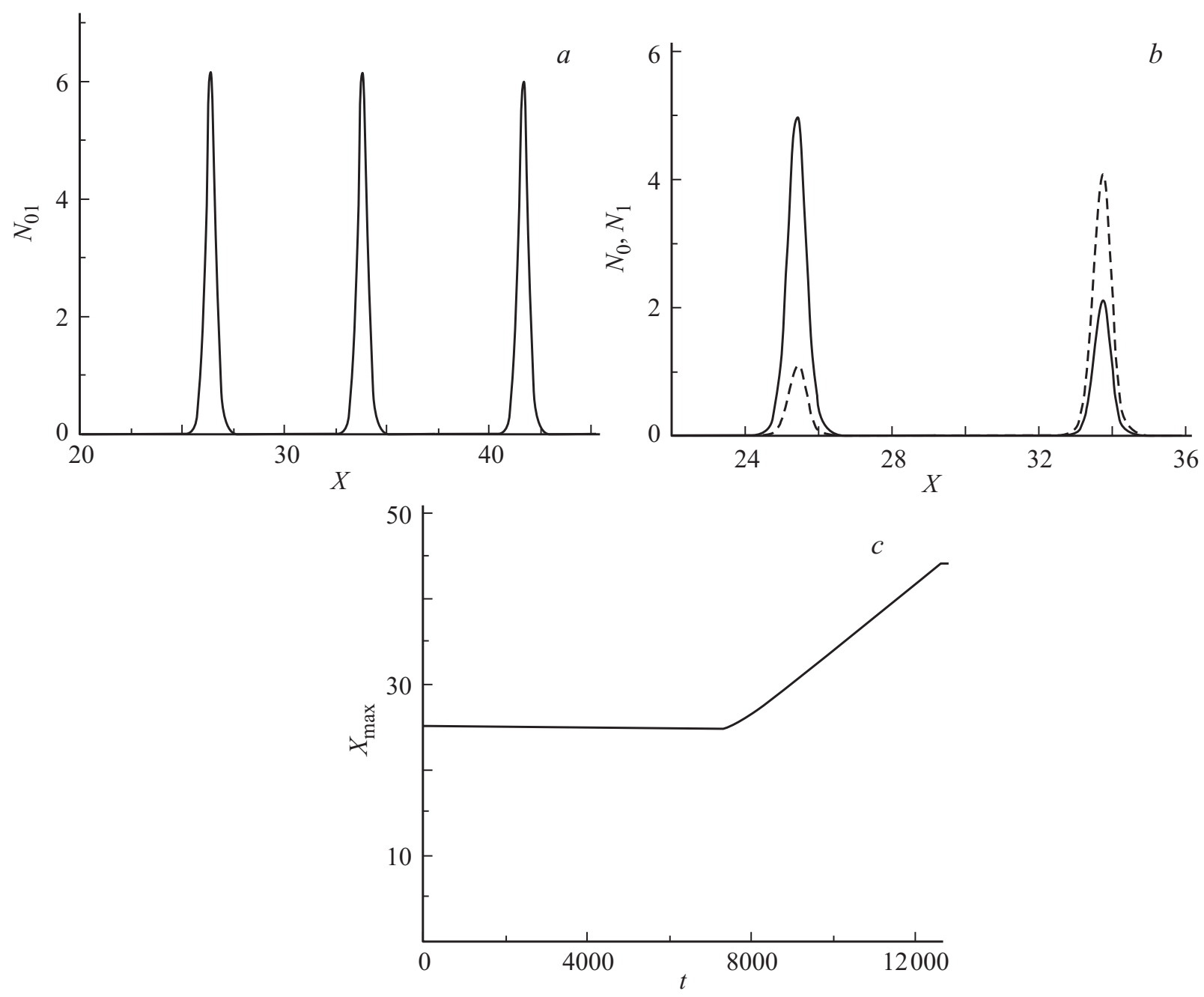

Рис. 3. Динамика пары синфазного и противофазного осциллонов при начальном совмещении профилей населенности: профили $(a)$ суммарной населенности для трех моментов времени $(t=8000$ для левого профиля, 10000 для среднего, 12000 для правого) и $(b)$ населенностей компонент для двух моментов времени $\left(t=7600\right.$ для левого профиля, 10000 для правого, $N_{0}-$ сплошные кривые, $N_{1}-$ пунктир), (c) траектория максимума суммарной населенности. Параметры: $w_{1}=w_{2}=0.5, \Delta x_{0}=0$.

о траекториях осциллонов, характеризуя положение осциллона координатой максимума общей населенности состояний. Другой исключительный случай относится к сценариям с достаточно большой скоростью сближения, когда взаимодействие заведомо слабое (критерий приведен ниже). В общем случае существенного перекрытия профилей осциллонов последовательное определение их индивидуальных траекторий невозможно. И даже после окончания такого перекрытия, когда осциллоны уже разошлись, затруднительно сказать, имело ли место их прохождение друг сквозь друга или отталкивание. В ряде случаев с определенной долей условности мы будем различить осциллоны и после столкновения по соотношению из максимальных населенностей. Это затруднительно при столкновении двух осциллонов вида (11) с одинаковыми ширинами и соответственно максимальными населенностями. В этом случае будем вводить в их параметры небольшие различия, которые не меняют сценария взаимодействия, но позволяют соотнести траектории осциллонов до и после столкновения.

\section{Столкновения синфазных осциллонов. Большая относительная скорость}

Для различения осциллонов максимумальную амплитуду (квадратный корень из населенности) исходно неподвижного осциллона выбираем меньшей, чем у налетающего на него со скоростью $V=10$ на 5\% (такое различие используется и далее в других вариантах столкновений). В этом варианте для любых моментов времени населенности нижнего и верхнего состояний БЭК совпадают: $n_{0}(x, t)=n_{1}(x, t)$. Поэтому система управляющих уравнений (4) сводится к единственному НУШ (9) или (10), т. е. исходная векторная система превращается в скалярную. Динамика профиля суммарной населенности БЭК показана на рис. 5. Как до (рис. 5, $a$ ), так и после столкновения (рис. 5, c) видны два 


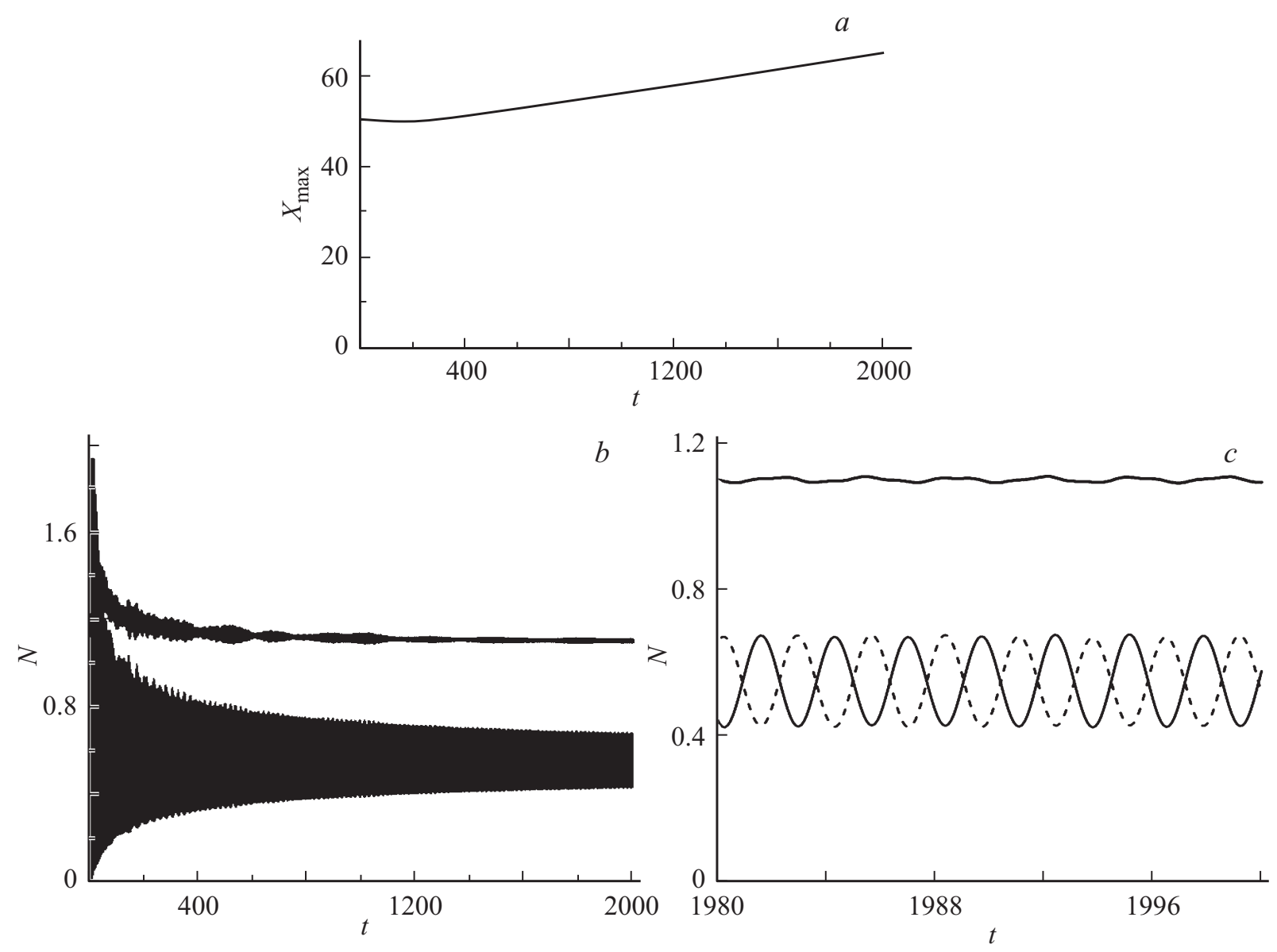

Рис. 4. Динамика пары синфазного и противофазного осциллонов при начальном пространственном расхождении профилей населенности: (a) траектория максимума суммарной населенности, $(b)$ установление со временем максимальной суммарной населенности осциллонов $N_{01}$ (верхняя кривая) и населенностей компонент $N_{0}=N_{1}$ (нижняя кривая), (c) установившаяся суммарная населенность осциллонов $N_{01}$ (верхняя кривая) и населенностей компонент $N_{0}$ (нижняя сплошная кривая) и $N_{1}$ (пунктир). Параметры: $w_{1}=w_{2}=1, \Delta x_{0}=0.4$.

отчетливых пика населенности, профиль населенностей в каждом из которых близок к описываемому соотношением (11). Существенное перекрытие этих профилей с временным формированием квазиинтерференционной структуры происходит только на коротком интервале времени около момента столкновения (рис. $5, b$ ).

На рис. 6, $a$ показаны траектории максимумов суммарной населенности двух уровней $n_{01}$ (которые в данном случае равнонаселены). Как указывалось выше, такие траектории теряют смысл в области перекрытия распределений населенности для сталкивающихся солитонов $(t \approx 1.4)$. Это затрудняет идентификацию солитонов после столкновения, т.е. соотнесение их с исходными. Однако в данном случае уходящий после столкновения осциллон движется с той же скоростью, что и налетающий, а также обладает несколько большим максимумом населенности (сравни рис. 5, $b, c$ ). Это позволяет в соответствии с приведенным выше условием слабости взаимодействия интерпретировать его как прохождение одного осциллона через другой с сохранением скорости, формы и числа атомов. Из рис. 6, $a$ следует также, что исходно неподвижный солитон при взаимодействии практически сохраняет неподвижность и взаимодействие не приводит к замедлению исходно движущегося осцилллона. На рис. $6, b$ показано, как максимальная суммарная населенность двух осциллонов после их столкновения, сопровождающегося подъемом максимума при одновременном пространственном сжатии осциллонов, возвращается к исходному значению. Укажем также, что оба осциллона после столкновения остаются синфазными. Напомним, что векторная природа осциллонов в этом варианте не проявляется.

\section{Столкновения синфазных осциллонов. Малая относительная скорость}

В этом варианте также сохраняется как синфазность осциллонов, так и равенство населенностей двух состояний БЭК: $N_{0}(x, t)=N_{1}(x, t)$. Поэтому здесь вновь не проявляется векторная природа осциллонов.

Как и в предыдущем случае, для различения („маркировки“) солитонов полагаем максимальную населен- 

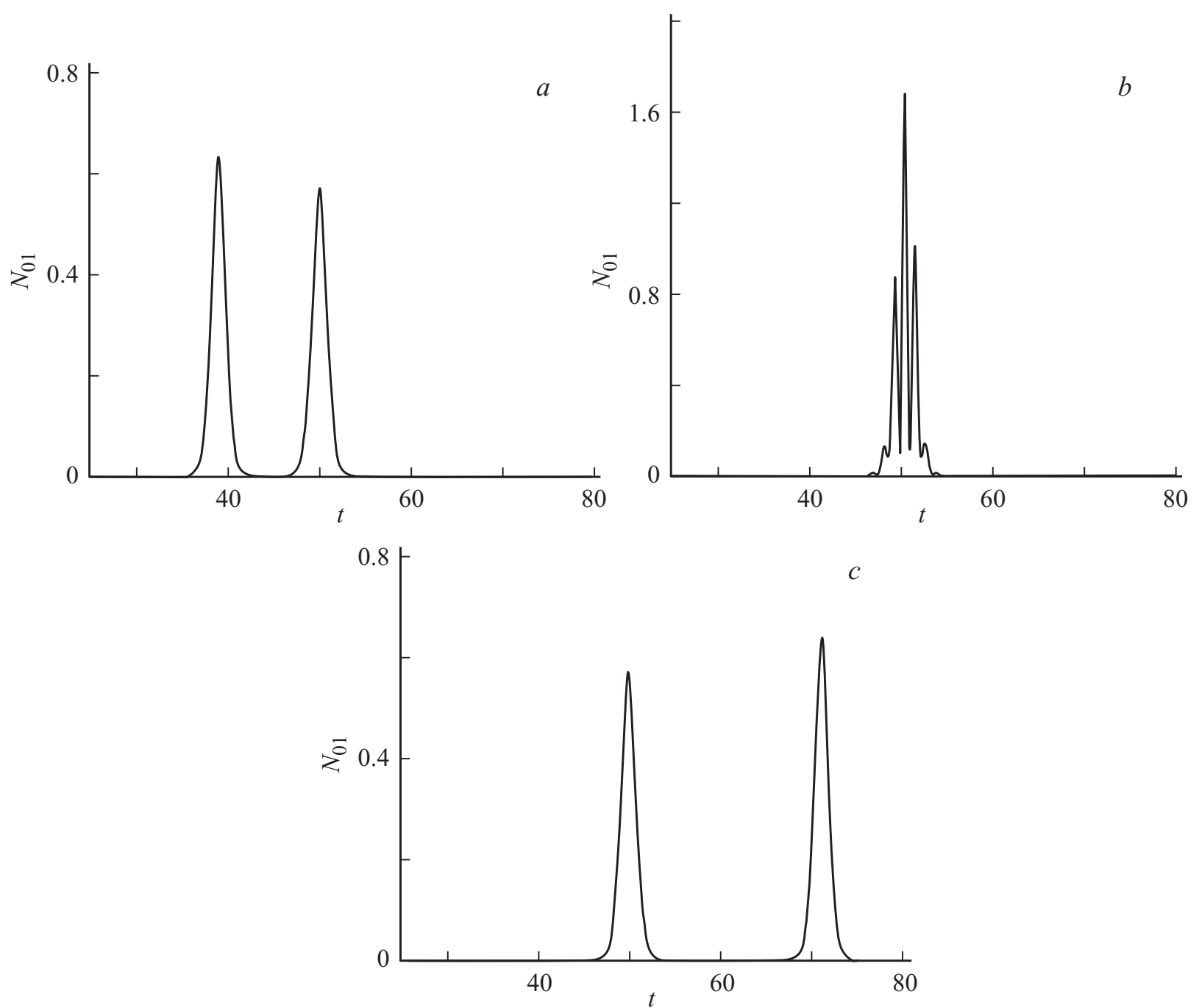

Рис. 5. Профили суммарной населенности двух уровней БЭК $N_{01}:(a)$ до столкновения $(t=0.1),(b)$ во время столкновения $(t=1.3)$, (c) после столкновения $(t=3.3)$ двух синфазных осциллонов, ширины исходных осциллонов $w_{1}=1, w_{2}=0.95$, начальная скорость сближения $V=10$.
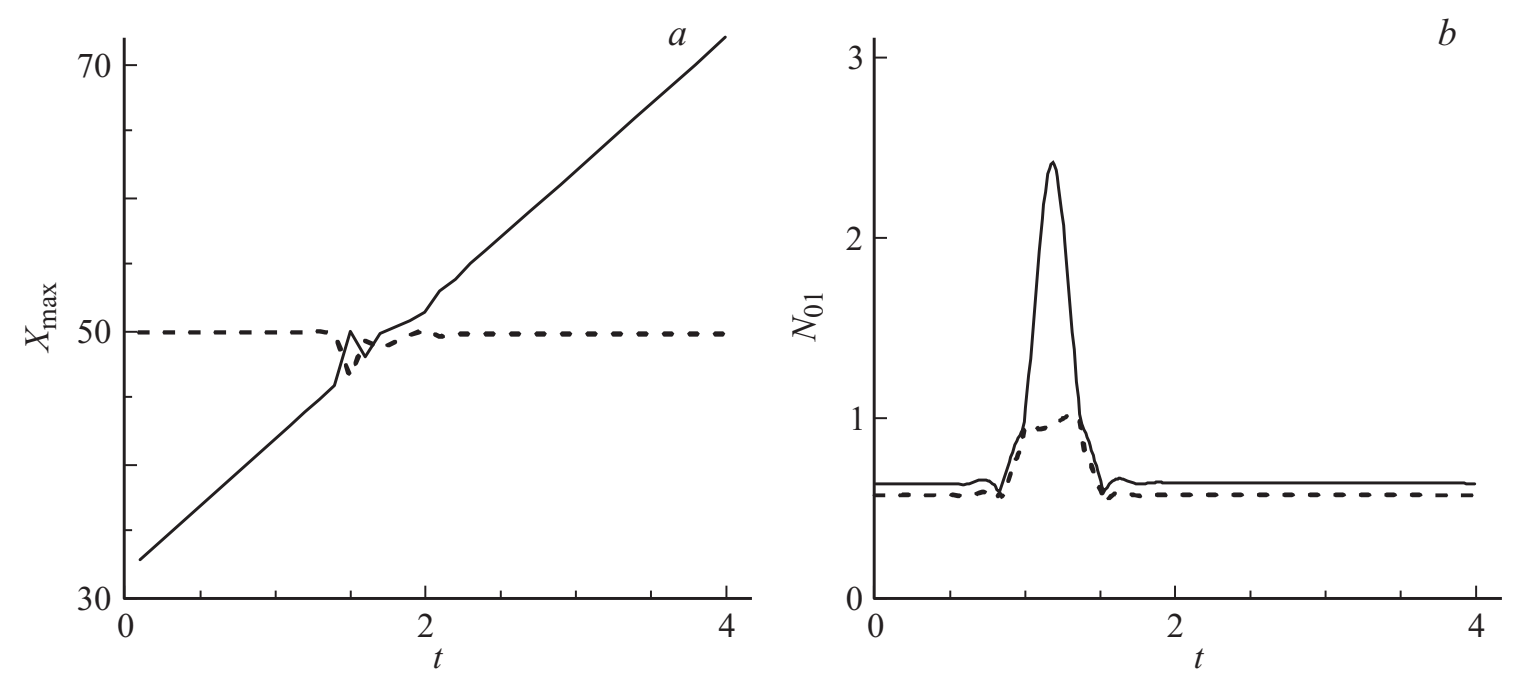

Рис. 6. (a) Траектории максимумов суммарной населенности и $(b)$ временная зависимость суммарной населенности для двух сталкивающихся синфазных осциллонов в условиях рис. 5 . 

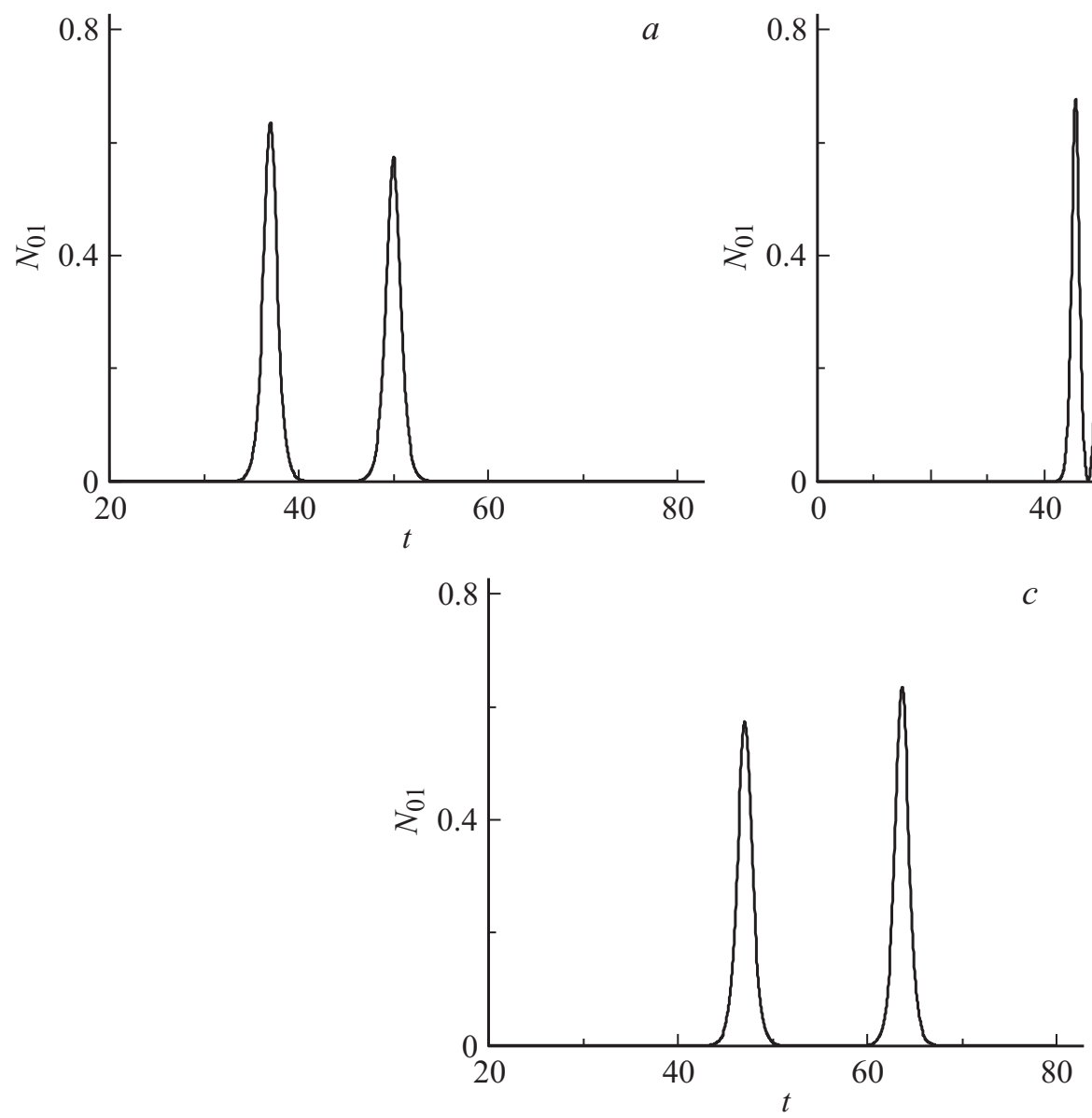

$b$

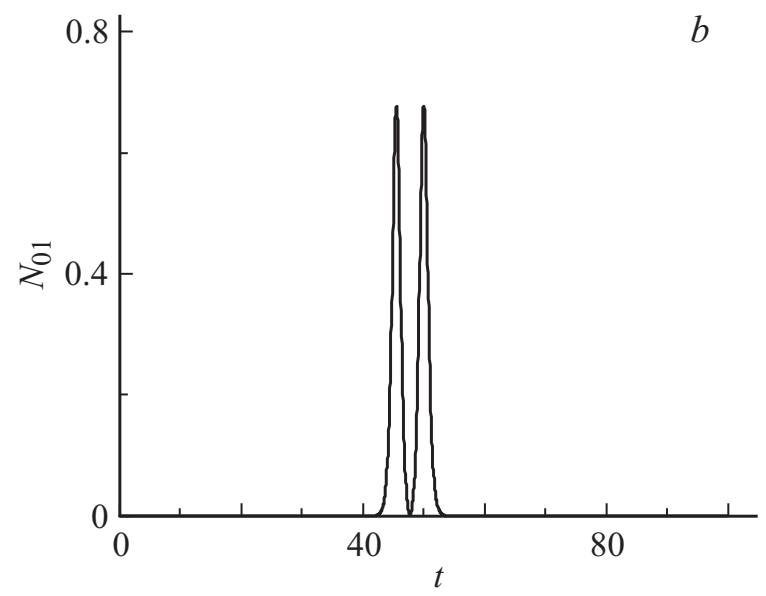



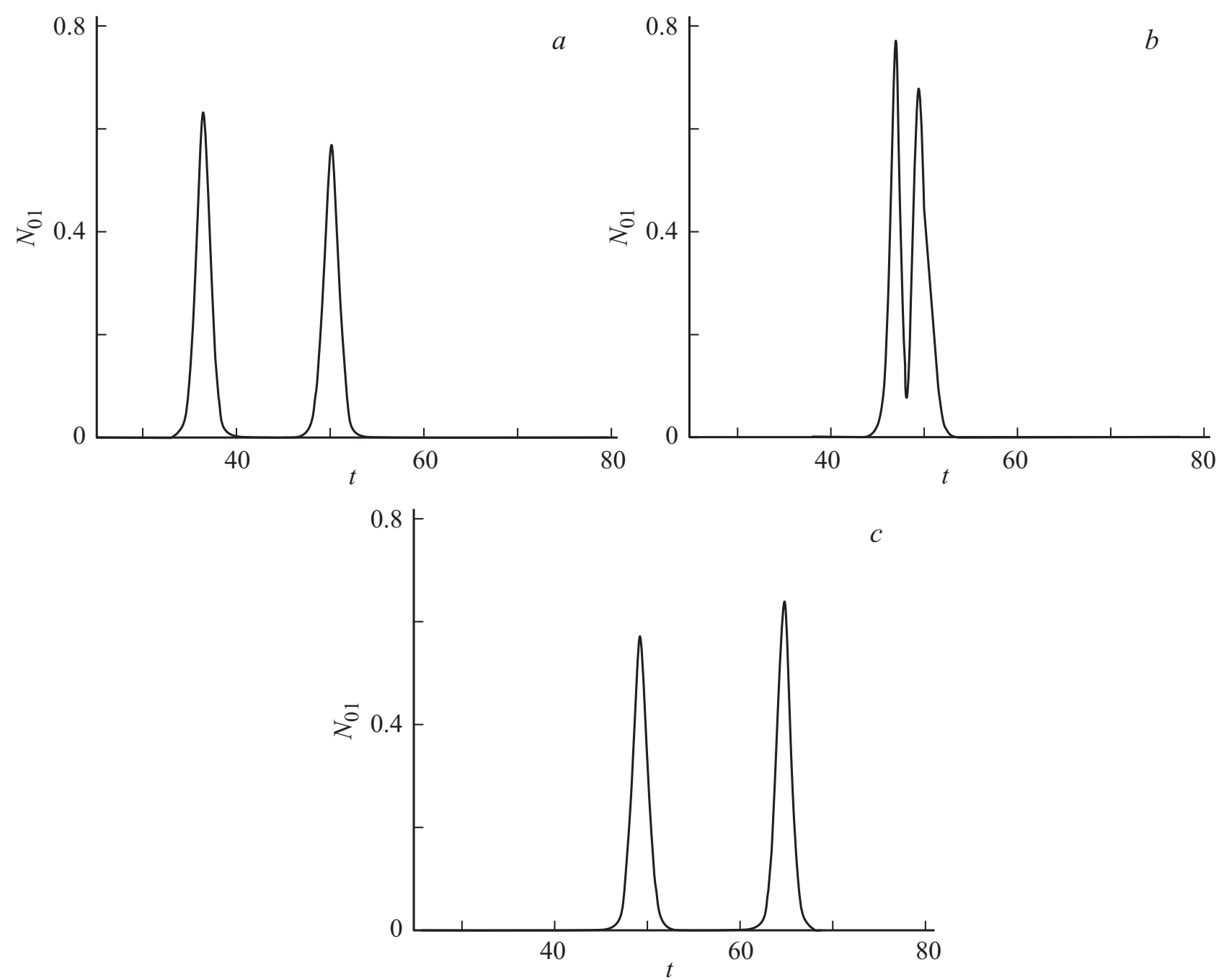

Рис. 9. То же, что на рис. 5, при скорости $V=4$. Моменты времени $t=0.1(a), 2.8(b), 7(c)$. Ширины осциллонов $w_{1}=1$, $w_{2}=0.95$.

нимальное расстояние исходно двигавшийся осциллон останавливается (при $t \approx 11$ ), после чего в движение с той же скоростью приходит другой осциллон. Помимо скорости, этот осциллон обладает еще одним признаком налетающего - несколько большей максимальной населенностью. Эти обстоятельства приводят к конфликту двух указанных выше критериев различения осциллонов: затрудняют интерпретацию столкновения. Действительно, при различении солитонов по их максимальной концентрации речь идет о продолжении движения исходно движущегося осциллона, что приводило бы к скачку его траектории в области наибольшего сближения (рис. 8, $a$, момент времени $t=16)$. Однако с большим основанием можно говорить о передаче его импульса (вместе с частью частиц) исходно неподвижному осциллону в той же области в условиях неперекрывания профилей населенности. Преимуществом второго варианта служит гладкость временной зависимости максимальной концентрации для двух осциллонов (рис. $8, b$ ).

\section{Столкновения синфазных осциллонов. Промежуточные значения скорости}

И в этом случае $(V=4)$ не нарушается равенство населенностей двух состояний БЭК: $N_{0}(x, t)=N_{1}(x, t)$, и осциллоны во время и после столкновения остаются синфазными. В профиле населенностей до момента столкновения (рис. 9, $a$ ) видны два четко различаемых пика, которые можно сопоставить осциллонам. В области столкновения (рис. 9, $b$ ) формируется промежуточная структура с резким сужением и ростом максимумов пиков. Затем от этой структуры отделяется и улетает осциллон, обладающий практически той же скоростью и максимальной населенностью, что и исходный налетающий. Другой осциллон с той же точностью остается неподвижным. Тем не менее как из рис. 9, так и из рис. 10, $a$, на котором представлены траектории двух главных максимумов суммарной населенности, а также из рис. $10, b$, на котором показана временная зависимость соответствующих значений населенности, нельзя 

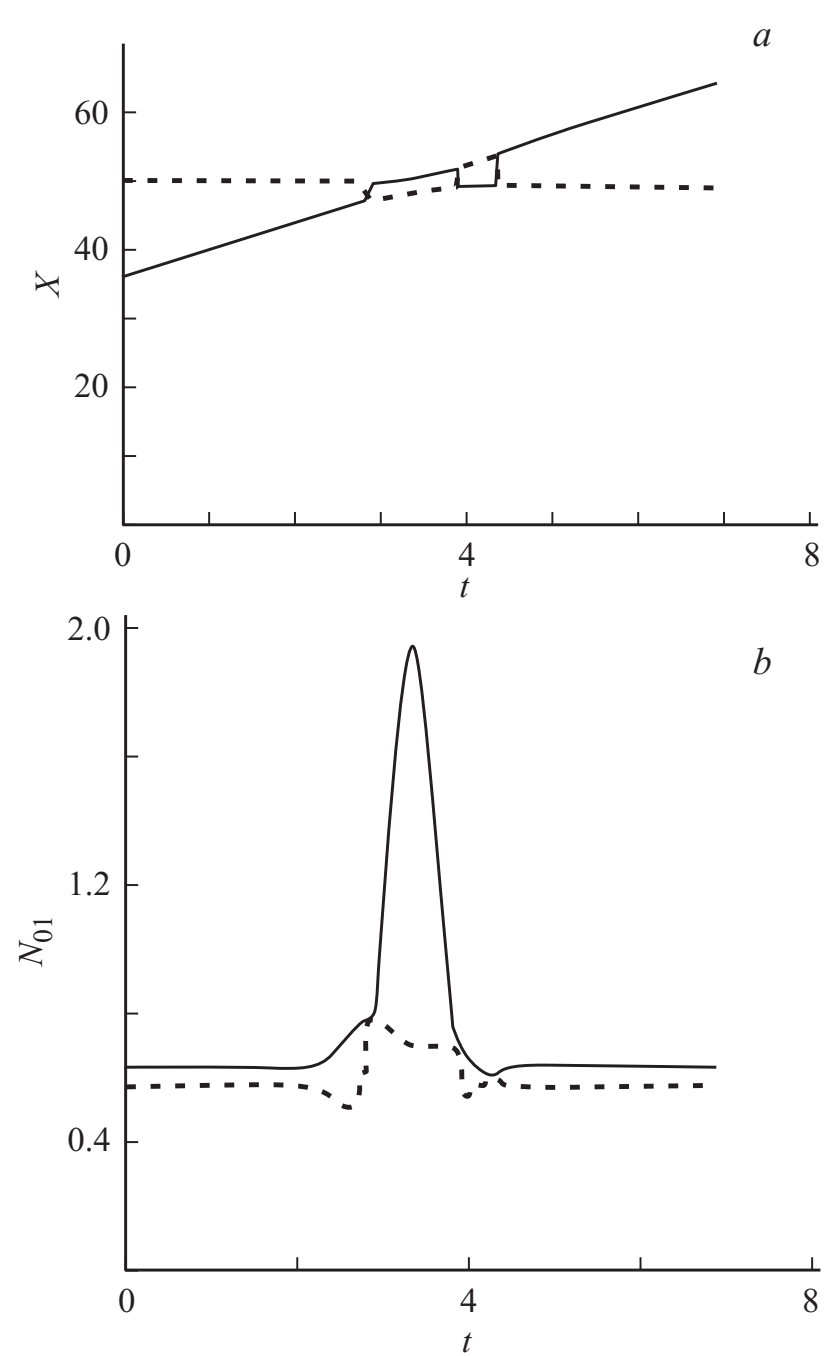

Рис. 10. То же, что на рис. 6, в условиях рис. 9.

причислить этот сценарий столкновения к прохождению или отталкиванию осциллонов.

Совершенно аналогичны сценарии столкновения двух противофазных осциллонов. Во всех случаях после взаимодействия осциллонов одинаковых типов они остаются фундаментальными (вида (11)) и их векторная природа при столкновениях не проявляется. Сравнение асимптотик траекторий (прямых) на рис. 6, $a$ и 8, $a$ показывает, что при больших скоростях сближения уходящий осциллон идет с задержкой (по сравнению со случаем отсутствия второго осциллона), а при малых скоростях - с опережением. Естественно, что при промежуточных значениях скорости и результат оказывается промежуточным.

\section{Столкновения синфазного и противофазного осциллонов. Большая скорость}

При взаимодействии осциллонов различных типов синфазного и противофазного - их векторная природа уже не может не проявляться в той или иной степени.
Не является исключением и случай большой скорости сближения, в котором столкновение вносит лишь слабые возмущения в характеристики осциллонов, ввиду чего мы не показываем рисунки, аналогичные рис. 5 и 6. Однако, как показывает рис. $11, a$, в области столкновения профили населенностей двух состояний БЭК оказываются сдвинутыми друг относительно друга, что и служит признаком векторности осциллонов. В то же время до и после столкновения эти состояния заселены
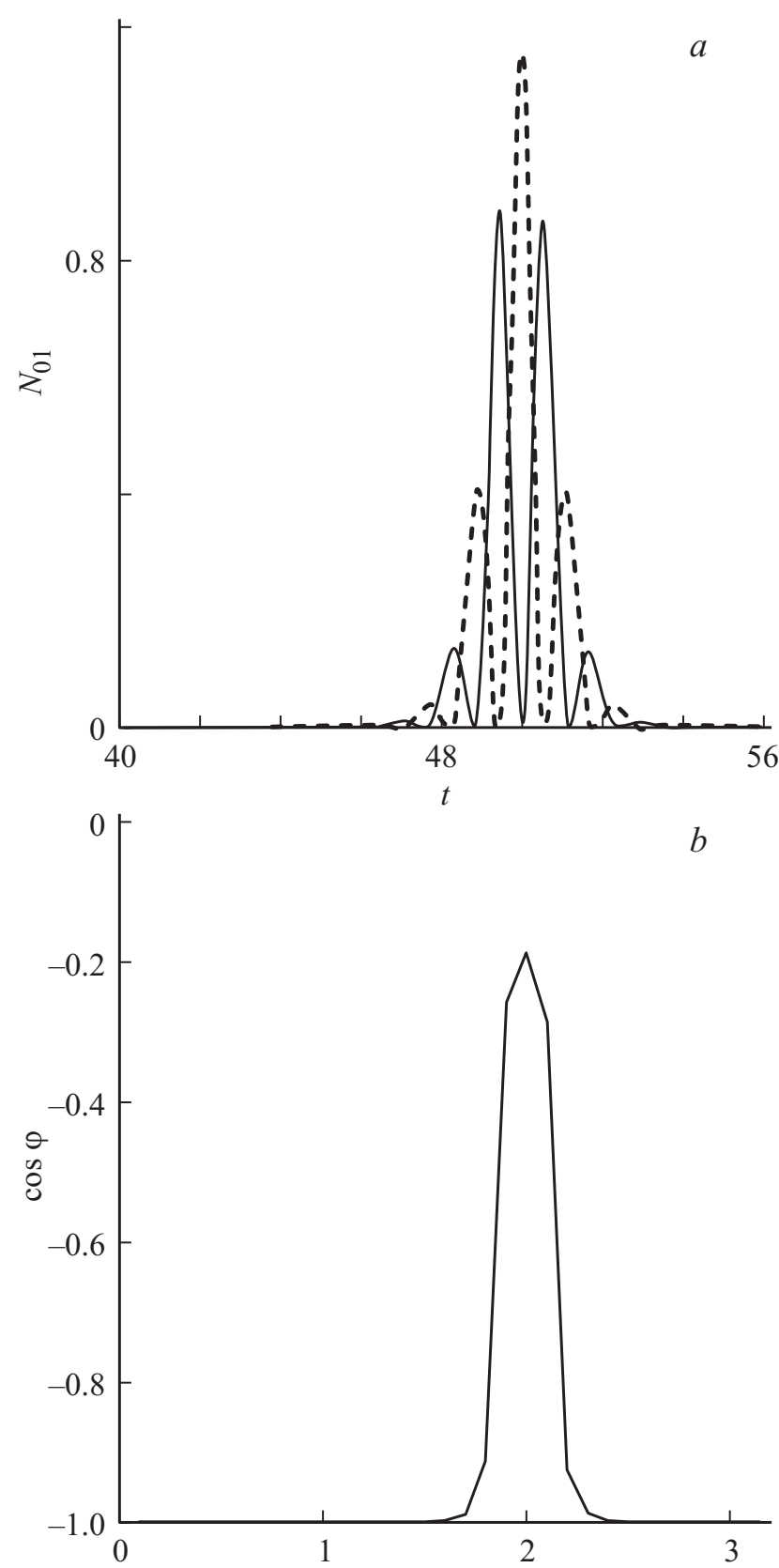

Рис. 11. (a) Профили населенности $N_{0}$ и $N_{1}$ двух состояний БЭК в момент столкновения синфазного и противофазного осциллонов. (b) Временная зависимость $\cos \varphi$, где $\varphi-$ разность фаз двух состояний, для „быстрого“ осциллона. Ширины осциллонов $w_{1}=1, w_{2}=0.95$, начальная скорость их сближения $V=10$. 


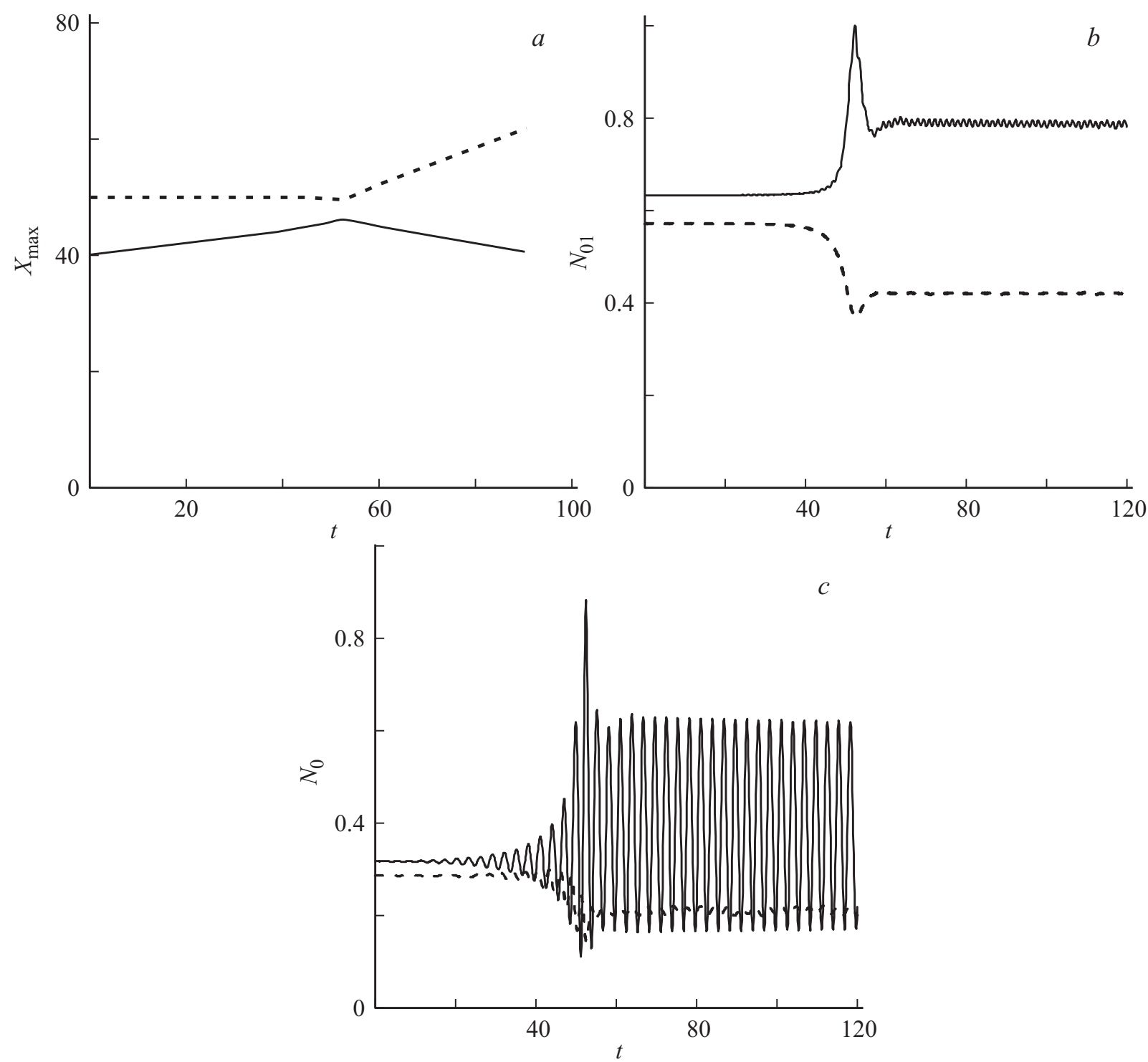

Рис. 12. Координаты максимумов суммарной населенности $(a)$, временная зависимость суммарной населенности $(b)$ и населенности основного состояния $(c)$ при столкновении со скоростью $V=0.1$ синфазного осциллона с неподвижным противофазным. Ширины осциллонов $w_{1}=1, w_{2}=0.95$.

практически одинаково. Разность фаз двух состояний $\varphi=\arg a_{1}-\arg a_{0}$ для быстро движущегося (в данном случае синфазного) осциллона также существенно отклоняется от исходной во время столкновения и возвращается к ней после столкновения (рис. $11, b)$.

\section{Столкновения синфазного и противофазного осциллонов. Малая скорость}

Этот вариант иллюстрируют рис. 12 и 13, полученные при начальной скорости сближения $V=0.1$. Динамику профилей суммарной населенности не приводим ввиду ее качественной аналогичности изображенной на рис. 7. Траектории максимумов населенностей состояний для двух осциллонов показаны на рис. 12,a. Сравнение этих траекторий с приведенными на рис. $8, a$ позволяет интер- претировать режим в конечном счете как отталкивание двух осциллонов, однако в рассматриваемом случае оба осциллона после столкновения двигаются с заметными скоростями. Непосредственно перед столкновением на рис. 12, a можно выделить кратковременный этап притяжения осциллонов. Столкновение приводит к передаче определенной части частиц от исходно неподвижного осциллона к исходно движущемуся (рис. 12, b). Кроме того, небольшая часть частиц от локализованных структур переходит в волны сплошного спектра. Наконец, после столкновения осциллоны перестают быть чисто синфазными или противофазными. Действительно, населенности двух состояний заметно различаются и, кроме того, они осциллируют со временем, в особенности для исходно движущегося осциллона (рис. 12,c). Осциллирует и разность фаз двух состояний для исходно движущегося 


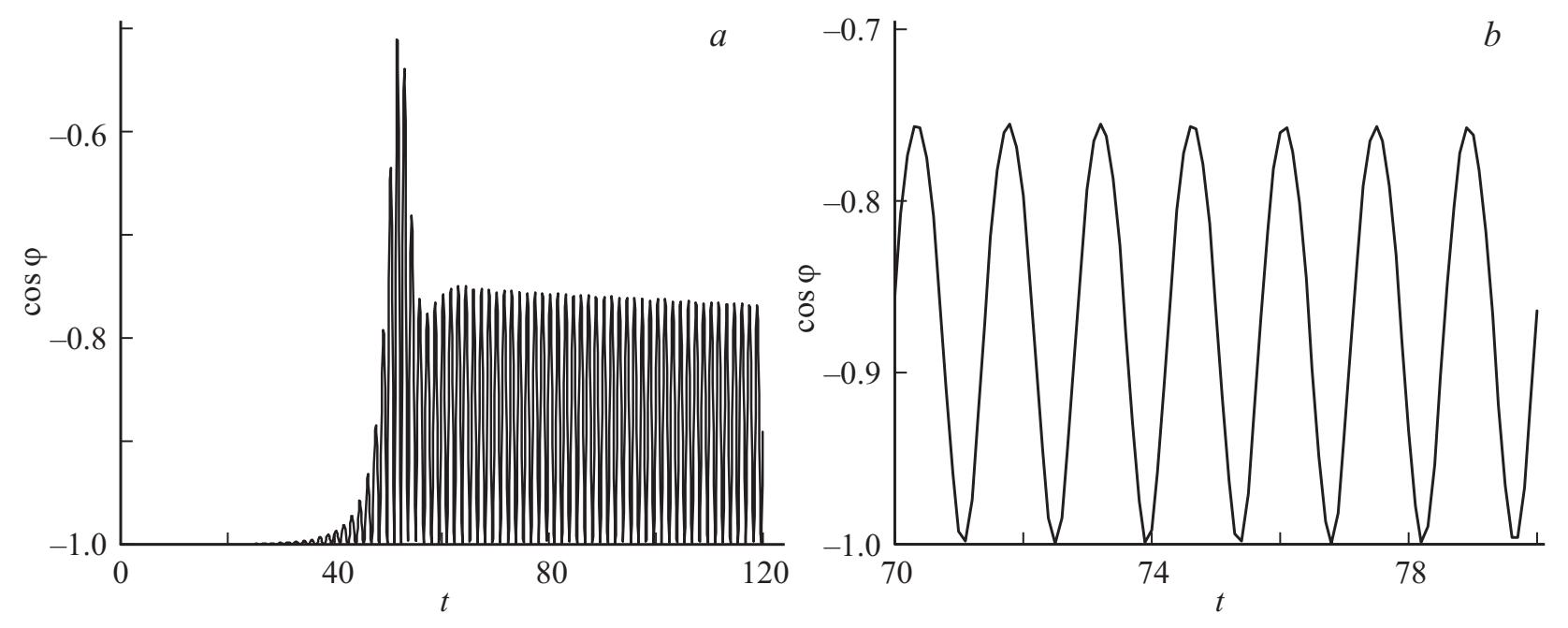

Рис. 13. Временная зависимость косинуса разности фаз двух состояний БЭК в двух разных масштабах для исходно движущегося осциллона в условиях рис. 12.
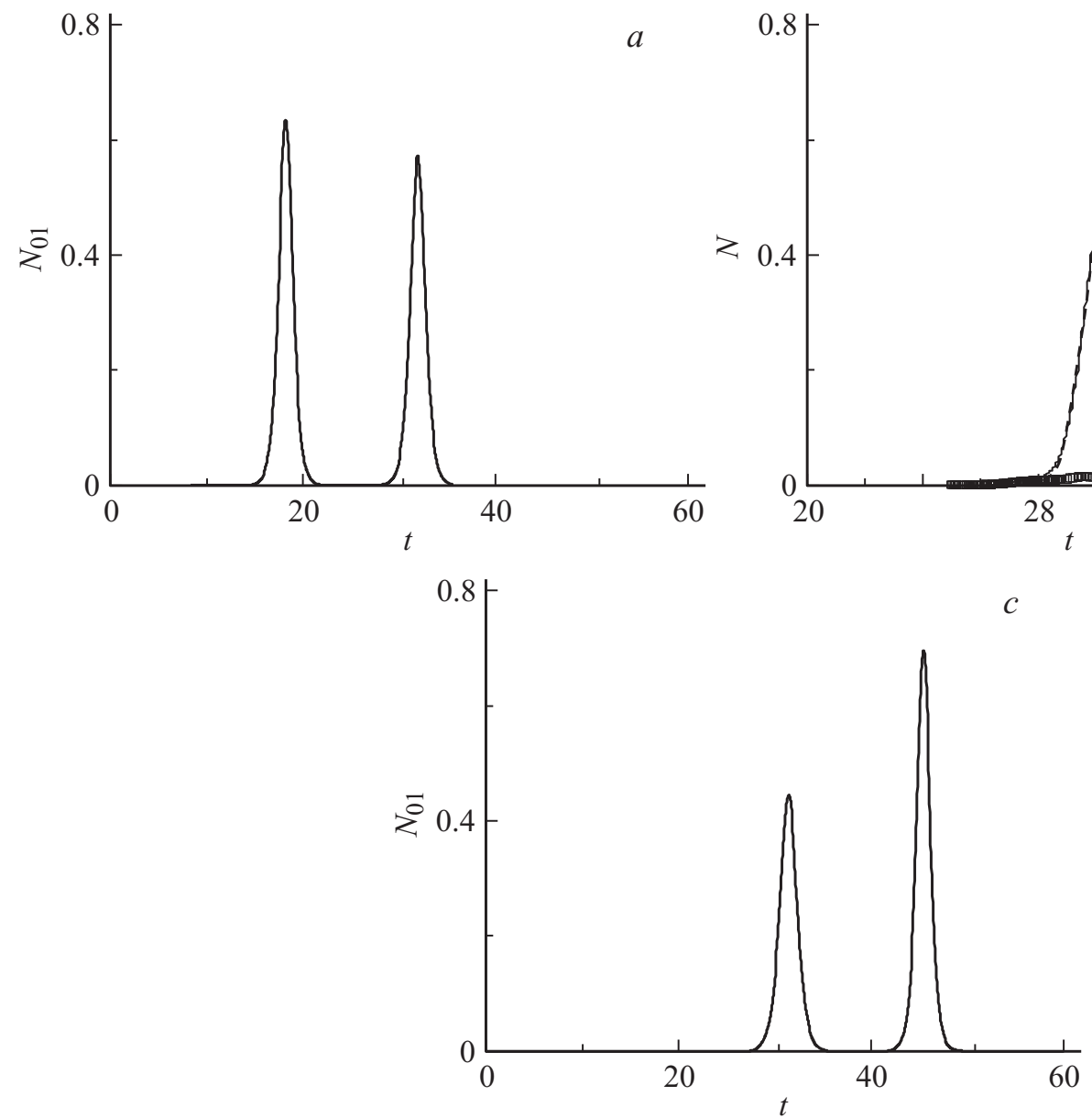

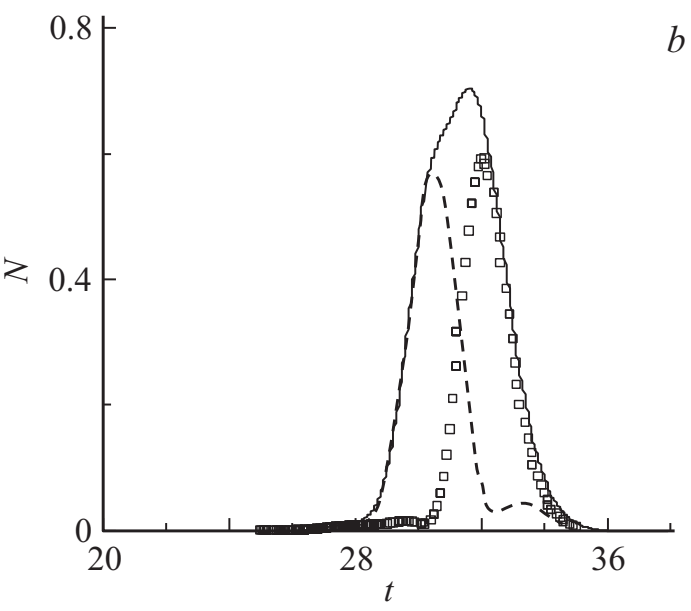

$b$

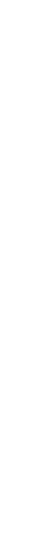



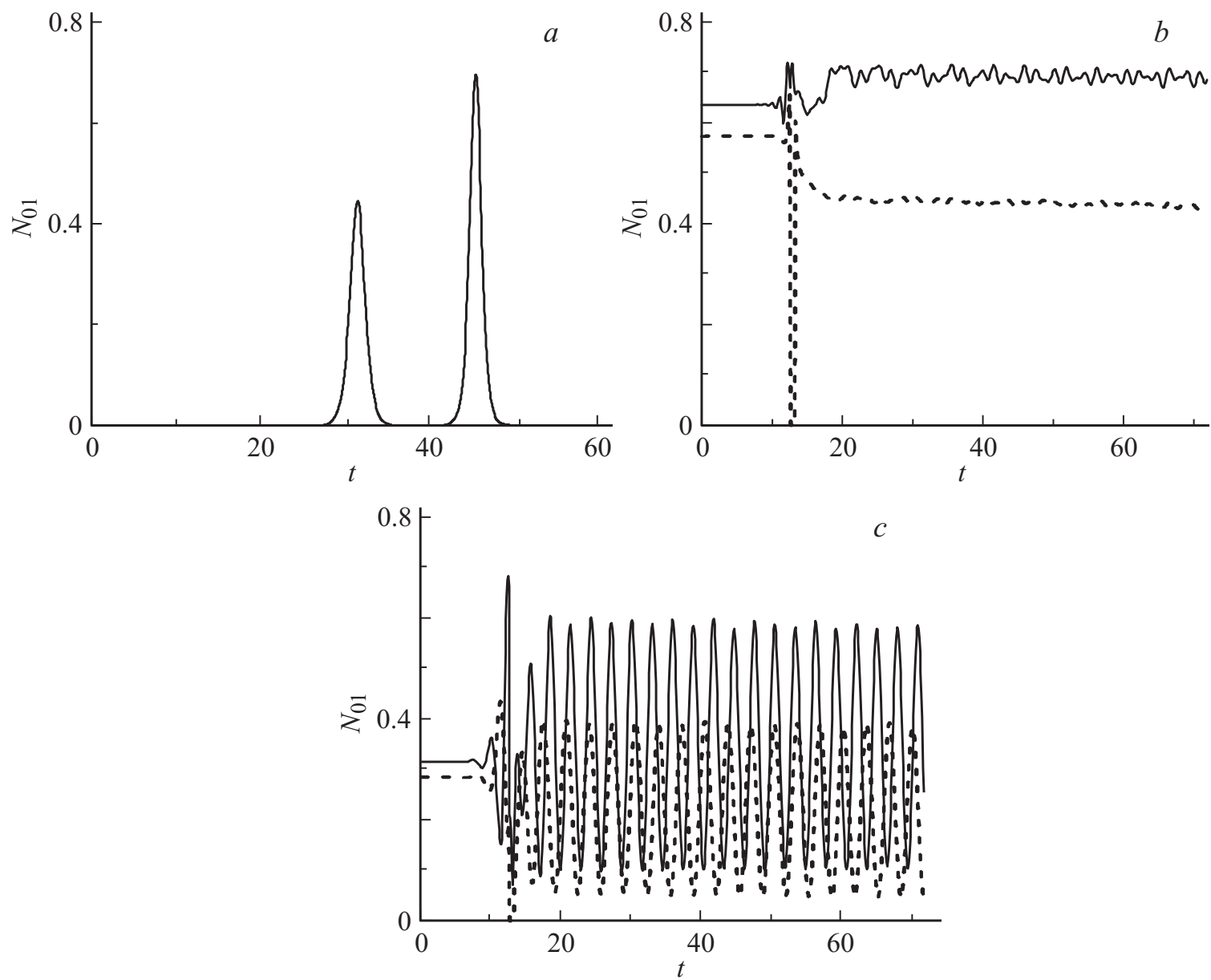

Рис. 15. Профили максимумов суммарной населенности обоих осциллонов $(a)$, временная динамика максимальной суммарной населенности обоих осциллонов $(b)$ и населенности $N_{0}(c)$. Сплошная кривая - налетающий осциллон, штриховая - изначально неподвижный. Ширины осциллонов $w=1, w=0.95$.

(синфазного) осциллона (рис. 13). Имеются основания считать, что в результате столкновения формируются долгоживущие векторные бризеры. Этот сценарий столкновений согласуется с представленным ранее в [1].

\section{Столкновения синфазного и противофазного осциллонов. Промежуточная скорость}

Расчеты проведены при скорости сближения осциллонов $V=1$. Динамика профилей суммарной населенности двух состояний БЭК, показанная на рис. 14, аналогична приведенной выше на рис. 9. Однако в данном случае достигается существенное различие населенностей двух состояний, как это видно из рис. $14, b$.

Траектории осциллонов и динамика максимальной населенности состояний показана на рис. 15. Применительно к рис. 15, $a$ напомним, что в области перекрытия осциллонов понятие их индивидуальных траекторий теряет смысл. После столкновения, которое в данном случае естественно относить к варианту прохождения, координата исходно неподвижного осциллона смещается и он приобретает небольшую скорость, равную 0.07. Как

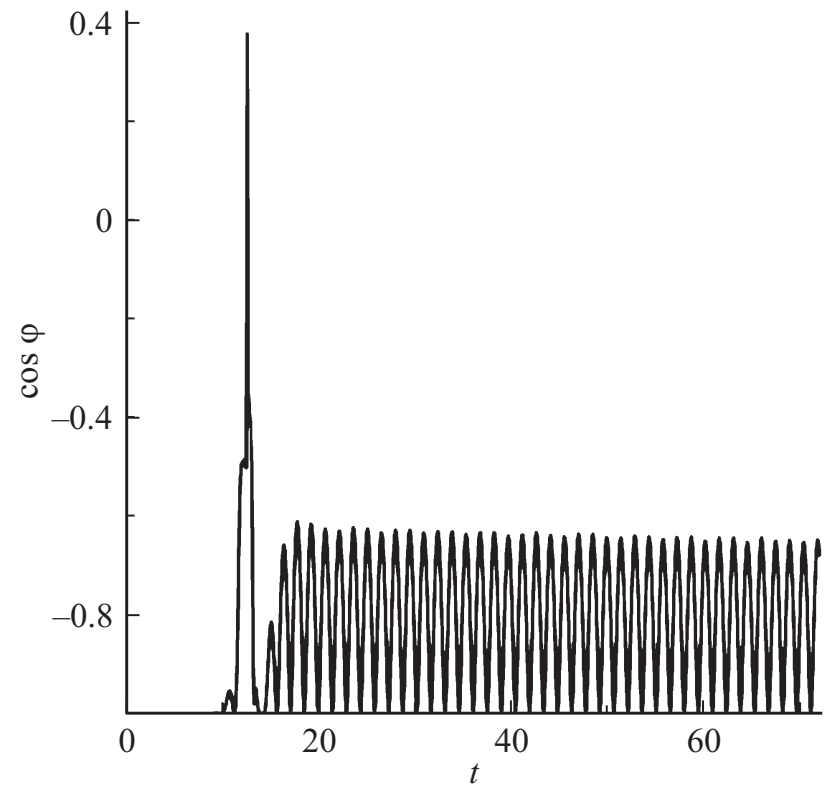

Рис. 16. Временная зависимость косинуса разности фаз двух состояний для исходно движущегося осциллона в условиях рис. 15. 
видно из рис. $15, b$ и $c$, в которых область перекрытия осциллонов также непредставительна, результатом столкновения служит трансформация осциллонов в бризеры с большой глубиной модуляции максимальных населенностей отдельных состояний БЭК и слабой модуляцией суммарной максимальной населенности. Разность фаз двух состояний для исходно движущегося осциллона после столкновения также сильно осциллирует (рис. 16). После столкновения происходит и некоторая потеря числа частиц в локализованных структурах вследствие их преобразования в волны сплошного спектра, уходящие на периферию системы.

\section{Обсуждение}

Таким образом, типичными связанными состояниями векторных осциллонов атомного БЭК являются симметричные или асимметричные бризеры. Вообще говоря, исключительно на основании численных расчетов затруднительно отличить осцилляции бризера от также долго продолжающихся переходных процессов, связанных с исключительно слабым затуханием внутренних мод консервативных солитонов различных типов [16-29]. Однако аргументом в пользу бризеров служит и то, что они сохраняются в ситуациях, описываемых единственным (скалярным) НУШ, в которых внутренних мод солитонов не имеется.

Проведенный анализ показывает, что при столкновении одинаковых осциллонов (двух синфазных или двух противофазных) оба они остаются фундаментальными и сохраняются как полное число частиц в них, так и разность фаз состояний в каждом осциллоне. В этом смысле векторный характер осциллонов, т.е. „векторные“ степени свободы существенно не проявляются. Столкновение синфазного и противофазного осциллонов при их большой относительной скорости ведет только к временному нарушению фазовых соотношений, а после окончания столкновения осциллоны вновь становятся фундаментальными. Однако при меньших относительных скоростях столкновение возбуждает новые степени свободы, связанные с векторностью осциллонов. Они превращаются в долгоживущие векторные бризеры с существенной временной модуляцией населенностей уровней и разности фаз состояний в результирующих локализованных структурах, что сопровождается уходом части частиц на периферию системы.

\section{Список литературы}

[1] Rosanov N.N., Sochilin G.B., Vinokurova V.D., Vysotina N.V.// Phil. Trans. R. Soc. A. 2014. V. 372. P. 20140012.

[2] Розанов Н.Н., Веретенов Н.А., Высотина Н.В., Нестеров Л.А., Федоров С.В., Шацев А.Н. // Опт. и спектр. 2015. T. 119. N 3. C. 388-396; Rosanov N.N., Veretenov N.A., Vysotina N.V., Nesterov L.A., Fedorov S.V., Shatsev A.N. // Opt. Spectrosc. 2015. V. 119. N 3. P. 363-370.
[3] Rosanov N. N. // Phys. Rev. A. 2013. V. 88. P. 063616.

[4] Розанов Н.Н., Сочилин Г.Б. // ЖЭТФ. 2014. Т. 145. № 1. C. 143-152; Rozanov N.N., Sochilin G.B. // JETP. 2014. V. 118. N 1. P. $124-132$.

[5] Rosanov N.N. // Phys. Rev. A. 2014. V. 89. P. 035601.

[6] Кившарь Ю.С., Агравал Г.П. Оптические солитоны. От волоконных световодов к фотонным кристаллам. М.: Физматлит, 2005. $647 \mathrm{c}$.

[7] Манаков С.В. // ЖЭТФ. 1974. Т. 67. № 8. С. 543-555.

[8] Питаевский Л.П. // УФН. 1998. Т. 168. № 4. С. 641-653.

[9] Moskalenko S.A., Snoke D.W. Bose-Einstein condensation of excitons and biexcitons and coherent nonlinear optics with excitons. Cambridge, UK: Cambridge University Press, 2000.

[10] Khamrakulov Kh.P. // Int. J. Mod. Phys. B. 2014. V. 28. P. 1450198.

[11] Benseghir A., Wan Abdullah W.A.T., Baizakov B.B., Kh. Abdullaev F.Kh. // Phys. Rev. A. 2014. V. 90. P. 023607.

[12] Высотина Н.В., Розанов Н.Н. // ЖЭТФ. 2016. Т. 150. № 1. C. 60-68; Vysotina N.V., Rosanov N.N. // J. Exp. Theor. Phys. 2016. V. 123. N 1. P. 51.

[13] Розанов Н.Н., Сочилин Г.Б. // Опт. и спектр. 2014. Т. 117. № 6. C. 883-887; Rosanov N.N., Sochilin G.B. // Opt. Spectrosc. 2014. V. 117. N 6. P. 855-860.

[14] Захаров В.Е., Манаков С.В., Новиков С.П., Питаевский Л.П. Теория солитонов: Метод обратной задачи. М.: Наука, 1980. $320 \mathrm{c}$.

[15] Захаров В.Е., Шабат А.Б. // ЖЭТФ. 1971. Т. 61. C. 118-134; Zakharov V.E., Shabat A.B. // Sov. Phys. JETP. 1972. V. 34. N 1. P. 62-69.

[16] Etrich C., Peschel U., Lederer F., Malomed B.A. // Phys. Rev. A. 1995. V. 52. P. R3444.

[17] Pelinovsky D.E., Buryak A.V., Kivshar Y.S. // Phys. Rev. Lett. 1995. V. 75. P. 591.

[18] Etrich C., Peshel U., Lederer F., Malomed B.A., Kivshar Y.S. // Phys. Rev. E. 1996. V. 54. P. 4321.

[19] Pelinovsky D.E., Afanasjev V.V., Kivshar Y.S. // Phys. Rev. E. 1996. V. 53. P. 1940.

[20] Pelinovsky D.E., Kivshar Yu.S., Afanasjev V.V. // Physica D. 1998. V. 116. P. 121.

[21] Artigas D., Torner L., Akhmediev N.N. // Opt. Commun. 1999. V. 162. P. 347.

[22] Крепостнов П.И., Попов В.О., Розанов Н.Н. // Опт. и спектр. 2000. Т. 89. № 6. С. 964-968; Krepostnov P.I., Popov V.O., Rosanov N.N. // Opt. Spectrosc. 2000. V. 89. N 6. P. 887.

[23] Etrich C., Lederer F., Malomed B.A., Peschel T., Peschel U. // Prog. Opt. 2000. V. 41. P. 485.

[24] Розанов Н.Н., Высотина Н.В., Владимиров А.Г. // ЖЭТФ. 2000. T. 118. № 12. C. 1307-1318; Rosanov N.N., Vysotina N.V., Vladimirov A.G. // JETP. 2000. V. 91. P. 1130.

[25] Rosanov N.N., Fedorov S.V., Kaliteevskii N.A., Kirsanov D.A., Krepostnov P.I., Popov V.O. // Nonlin. Opt. 2000. V. 23. P. $221-238$.

[26] Витенберг А.Б., Крепостнов П.И., Попов В.О., Розанов Н.Н. // Опт. и спектр. 2002. Т. 92. № 4. С. 603-607; Vitenberg A.B., Krepostnov P.I., Popov V.O., Rosanov N.N. // Opt. Spectrosc. 2002. V. 92. P. 550.

[27] Rosanov N.N., Vladimirov A.G., Skryabin D.V., Firth W.J. // Phys. Lett. A. 2002. V. 293. P. 45.

[28] Rosanov N.N., Krepostnov P.I., Popov V.O. // Chaos. 2003. V. 13. N 2. P. 791-799.

[29] Крепостнов П.И., Попов В.О., Розанов Н.Н. // ЖЭТФ. 2004. T. 126. № 2. C. 315-322; Krepostnov P.I., Popov V.J., Rozanov N.N. // JETP. 2004. V. 99. N 2. P. 279-285. 